\title{
Technè
}

La science au service de l'histoire de l'art et de la préservation des biens culturels

42 | 2015

Science et conservation

\section{Le Portrait d'Antoine de La Roque par Antoine Watteau (Tokyo, Fuji Art Museum) est-il un tableau à deux mains?}

Was another painter involved in the Portrait of Antoine de La Roque by Antoine Watteau (Fuji Art Museum, Tokyo)?

Bruno Mottin, Florence Raymond et Thomas Calligaro

\section{OpenEdition}

Journals

Édition électronique

URL : http://journals.openedition.org/techne/6960

DOI : $10.4000 /$ techne.6960

ISSN : 2534-5168

Éditeur

C2RMF

Édition imprimée

Date de publication : 1 décembre 2015

Pagination : 72-83

ISBN : 978-2-7118-6249-8

ISSN : 1254-7867

\section{Référence électronique}

Bruno Mottin, Florence Raymond et Thomas Calligaro, « Le Portrait d'Antoine de La Roque par Antoine Watteau (Tokyo, Fuji Art Museum) est-il un tableau à deux mains ?», Technè [En ligne], 42 | 2015, mis en ligne le 01 décembre 2015, consulté le 11 mars 2021. URL : http://journals.openedition.org/techne/ 6960 ; DOI : https://doi.org/10.4000/techne.6960

\section{(c) (†) $\ominus$}

La revue Technè. La science au service de l'histoire de l'art et de la préservation des biens culturels est mise à disposition selon les termes de la Licence Creative Commons Attribution - Pas d'Utilisation Commerciale - Pas de Modification 4.0 International. 


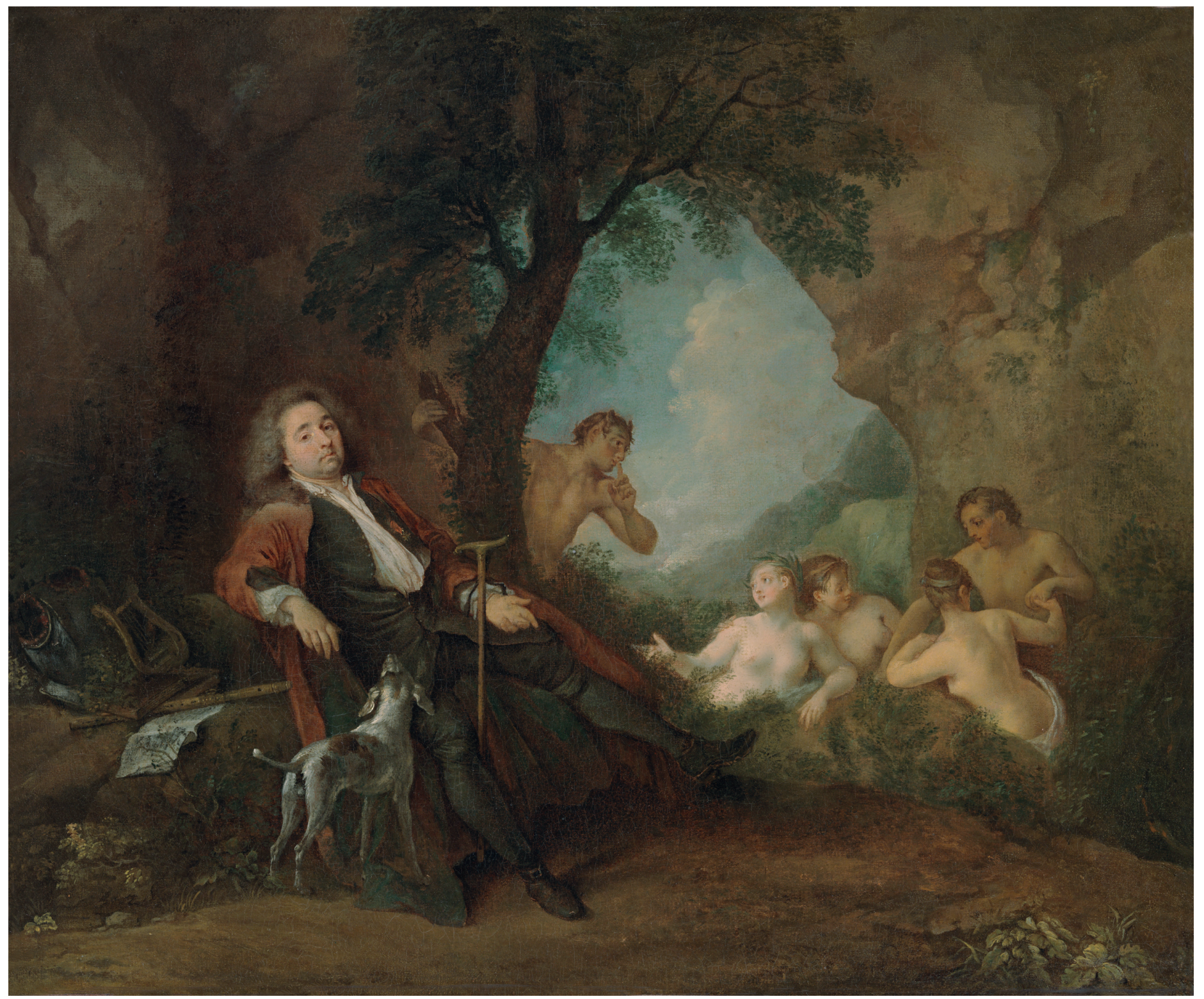

Fig. 1. Antoine Watteau et Nicolas Lancret? Portrait d'Antoine de La Roque, Tokyo, Fuji Art Museum. (c) C2RMF/Laurence Clivet. 
Bruno Mottin

Florence Raymond

Avec la collaboration

de Thomas Calligaro

\section{Le Portrait d'Antoine de La Roque par Antoine Watteau (Tokyo, Fuji Art Museum) est-il un tableau à deux mains ?}

Was another painter involved in the Portrait of Antoine de La Roque by Antoine Watteau (Fuji Art Museum, Tokyo)?
Résumé. Le Portrait d'Antoine de La Roque (Tokyo, Fuji Art Museum) est généralement considéré comme une cuvre d'Antoine Watteau et a été gravé sous ce nom par François-Bernard Lépicié, vers 1734, pour le Recueil Jullienne. Les historiens d'art s'interrogent cependant depuis quelques années sur une possible contribution de Nicolas Lancret à son exécution. L'étude réalisée en 2013 au Centre de Recherche et de Restauration des Musées de France (C2RMF) montre que la genèse de ce tableau est plus complexe encore. Seule la tête de La Roque semble pouvoir être attribuée à Watteau, alors que le reste de la partie centrale de la composition serait plutôt de Lancret. De plus, la composition était initialement plus petite et a été agrandie à deux reprises, d'abord par une mise à l'ovale, puis par une mise au rectangle qui lui a donné un aspect identique à la gravure. Pour cette démonstration, différentes techniques d'imagerie ont été exploitées, notamment l'imagerie par spectrométrie de fluorescence X, qui permet de cartographier les éléments chimiques présents sur l'œuvre.

Mots-clés. Antoine Watteau, Nicolas Lancret, FrançoisBernard Lépicié, Antoine de La Roque, changements de format, imagerie scientifique, imagerie par spectrométrie de fluorescence X.
Abstract. The Portrait of Antoine de La Roque (Fuji Art Museum, Tokyo) is generally regarded as one of Antoine Watteau's works and was engraved under his authorship, in about 1734, by François-Bernard Lépicié, for the Recueil Jullienne. For the past few years, however, art historians have been wondering whether Nicolas Lancret possibly contributed to its execution. A study carried out in 2013 at the Centre de Recherche et de Restauration des Musées de France (C2RMF) showed that the genesis of the painting was even more complex. Only La Roque's head can apparently be attributed to Watteau. The rest of the central part of the composition is now thought to have been painted by Lancret. Moreover, the composition was initially smaller and was enlarged twice, first when it was arranged in an oval format, then when it was altered into a rectangular format, identical to that of the engraving. Different imaging techniques were used to obtain these findings, namely X-ray fluorescence imaging, which enabled researchers to map the chemical elements present in the painting.

Keywords. Antoine Watteau, Nicolas Lancret, François-Bernard Lépicié, Antoine de La Roque, changes of format, scientific imaging, X-ray fluorescence imaging.
Le Portrait d'Antoine de La Roque ${ }^{1}$ (fig. 1) est une œuvre emblématique d'Antoine Watteau (1684-1721). Peint vers 1715, c'est l'un des rares portraits réalisés par ce maître. Il représente l'un des proches du peintre, Antoine de La Roque (1672-1744), personnage important du milieu culturel de la Régence qui est figuré dans une attitude pensive et une tenue négligée au milieu de personnages mythologiques. Conservé par les héritiers du modèle, ce tableau n'a longtemps été connu des spécialistes que grâce à la gravure de François-Bernard Lépicié (1698-1755), publiée vers 1734 pour le Recueil Jullienne. Vendu par les héritiers de La Roque au début du Xx ${ }^{\mathrm{e}}$ siècle, il a été acquis en 1988 par le Tokyo Fuji Art Museum². Sa redécouverte a toutefois jeté le trouble parmi les spécialistes du peintre. Alors qu'il ne connaissait l'œuvre que par une photographie, Pierre Rosenberg a noté dès 1984 que les faunes et nymphes de l'arrière-plan étaient davantage dans le style de Lancret que de Watteau, ce qui l'amena à suggérer que l'œuvre pouvait être un tableau à deux mains ${ }^{3}$. Le doute n'a pas été éclairci depuis : en 2013, l'un des auteurs de ces lignes a préféré présenter l'œuvre sous une double attribution, à Antoine Watteau et Nicolas Lancret, lors de l'exposition Antoine Watteau (1684-1721), La Leçon de musique ${ }^{4}$ organisée au Palais des Arts de Bruxelles.

On sait gré aux responsables du Tokyo Fuji Art Museum et du Palais des Beaux-Arts de Bruxelles (BOZAR) d'avoir accepté de soumettre cette œuvre à l'étude scientifique du Centre de Recherche et de Restauration des Musées de France (C2RMF), à la suite de l'exposition bruxelloise, pour tenter de clarifier ce problème d'attribution. Nous avons disposé du tableau pendant environ un mois, pour l'examiner sous loupe binoculaire, réaliser un dossier d'imagerie scientifique (photographies sous différentes lumières, réflectographie infrarouge, radiographie) et procéder à une série d'analyses non invasives, c'est-à-dire sans prélèvement ni risque pour l'œuvre,

Bruno Mottin, conservateur en chef au C2RMF (bruno.mottin@culture.gouv.fr). Florence Raymond, attachée de conservation au Palais des Beaux-Arts de Lille (fraymond@mairie-lille.fr). Avec la collaboration de Thomas Calligaro, ingénieur de recherche au C2RMF (thomas.calligaro@culture.gouv.fr). 
afin d'en mieux connaître les constituants. Nous avons également pu nous appuyer sur les abondantes données réunies par le C2RMF sur la technique de Watteau et de ses émules, dont les principaux résultats ont été présentés en 2009-2010 dans un numéro spécial de la revue Techne ${ }^{5}$. Cet article est donc le fruit du travail d'une équipe, aidée par de nombreux connaisseurs de la peinture française du XVIII ${ }^{\mathrm{e}}$ siècle avec lesquels nous avons pu échanger ${ }^{6}$.

\section{Un tableau en forme de Conversation Piece}

Le tableau est peint sur une toile de dimensions moyennes, de $36 \mathrm{~cm}$ de haut par $55 \mathrm{~cm}$ de long. Antoine de La Roque à marcher, son chien à ses côtés. L'homme est singulier ; fils d'une famille de commerçants marseillais spécialisés dans le négoce avec l'Orient, il a préféré la carrière des armes au commerce en s'engageant dans la Garde Royale. En 1709, un boulet de canon lui a fracassé la jambe gauche à la bataille de Malplaquet, ce qui l'a forcé à s'arrêter. Le tableau le représenterait environ six ans après l'accident, vers $1715^{7}$. Il est vêtu d'un costume civil, à moitié boutonné, sur lequel il a enfilé un manteau dont les épaules sont tachées par la perruque poudrée. En souvenir de ses faits d'armes, il arbore la médaille au ruban rouge de l'ordre de Saint-Louis et désigne de la main gauche sa jambe mutilée. Depuis son accident, il s'est tourné vers d'autres activités : sa cuirasse, posée à gauche, est couverte par une partition et par des instruments de musique qui font allusion aux livrets d'opéras qu'il a écrits en 1713, pour une Médée et Jason, et en 1715, pour une Théonoé, composées par Joseph-François Salomon. Le lieu où se tient La Roque est difficile à caractériser : l'arrière-plan est fermé par une falaise rocheuse percée d'une ouverture découvrant un vaste paysage verdoyant. La Roque ne peut être assis à l'intérieur d'une grotte, car un arbre et des buissons s'y développent. Cette paroi constitue peut-être une sorte de frontière entre deux mondes, à la lisière desquels s'ébat un groupe de faunes et de nymphes à demi-nus. L'un des personnages, dissimulé derrière un arbre, vient de remarquer La Roque et cherche à imposer le silence au reste du groupe. L'œuvre est doucement poétique et le visage de La Roque exprime une mélancolie qui est typique de Watteau. Elle unit de façon étrange l'art du XVII ${ }^{\mathrm{e}}$ siècle, où l'aristocratie s'est volontiers fait dépeindre sous les traits de personnages mythologiques, et celui du XVIII $^{\mathrm{e}}$ siècle, où les personnages sont mis en scène dans un cadre naturel, vêtus familièrement, à la manière des Conversation Pieces de la peinture anglaise.

L'œuvre ne fait aucune allusion à la principale fonction occupée par La Roque à ce moment. Depuis 1677, il est pourtant membre-fondateur de la revue Le Nouveau Mercure, qui a succédé au Mercure Galant ${ }^{8}$. Il écrit fréquemment dans cette revue qui donne le ton de l'activité culturelle parisienne et dont il devient le directeur en 1724, année où elle prend le titre de Mercure de France. La Roque est également secrétaire du Conseil du dedans sous la Régence et demeure alors chez l'abbé Fraguier, qui est l'auteur d'un texte rendant hommage à Watteau après sa mort ${ }^{9}$.

Antoine de La Roque est un proche de Watteau, sans être son ami. Les deux hommes ont pu se rencontrer dès 1709 à Valenciennes, quand La Roque se rétablissait de sa blessure alors que l'artiste séjournait dans sa famille ${ }^{10}$. Il semble toutefois plus vraisemblable que leur rencontre se soit produite à Paris, dans le monde du théâtre pour lequel La Roque écrivait et Watteau peignait. Un Portrait présumé de La Roque dessiné par Watteau, conservé au Fitzwilliam Museum de Cambridge $^{11}$, pourrait témoigner de cette rencontre, bien que la ressemblance de l'homme à béquilles représenté sur le dessin avec le portrait de Tokyo ne soit pas évidente. La Roque est l'auteur de la première notice nécrologique détaillée consacrée à Watteau, publiée dans le numéro d'août 1721 du Mercure $^{12}$, et annonce régulièrement dans sa revue les progrès du Recueil Jullienne, monumental recueil de gravures réalisées d'après les peintures et dessins de Watteau, orchestré par Jean de Jullienne (1686-1766) entre 1723 et $1735^{13}$. Trois peintures et trente-neuf dessins de Watteau figurent dans le catalogue de sa vente après décès, alors que le présent tableau en est absent $^{14}$.

\section{La gravure du Recueil Jullienne}

La gravure du Portrait d'Antoine de La Roque figure dans le quatrième volume du Recueil Jullienne, deuxième tome de l'Euvre gravé ${ }^{15}$ consacré à la reproduction des peintures (fig. 2). Cette estampe tient une place toute particulière dans la carrière du graveur, François-Bernard Lépicié, car elle lui servit de morceau de réception à l'Académie ${ }^{16}$. CEuvre appliquée, destinée à convaincre ses pairs, elle est fidèle à l'œuvre étudiée ici : inversée par rapport au tableau, elle en reprend le cadrage et n'en diffère que dans de légers détails tels que l'orientation de la cuirasse ou l'épaisseur du haut de l'arbre. La lettre porte les noms du peintre et du graveur, «Watteau pinx./Lepicié Sculp.», et fait en quelques lignes l'éloge du modèle : «ANTOINE DE LA ROQUE / Ecuyer, Chevalier de l'ordre Royal et Militaire de St Louis, gratifié par sa Majesté du Brevêt et Privilège du Mercure de France. / Victime du Dieu Mars; les Filles de Mémoire, Occupent à présent son cœur et son Esprit. / Il a combattu pour la Gloire,/ Et c'est pour elle qu'il écrit. / A Paris/avec privilège du Roy. » Le tableau est donc un hommage au héros de Malplaquet qui a désormais tourné son esprit vers les filles de Mémoire, c'est-à-dire les Muses. Ces dernières sont évoquées au moyen des livres et des instruments de musique posés près de La Roque, et non par les personnages de l'arrière-plan qui n'en ont aucun attribut.

Si la gravure du Recueil Jullienne donne clairement l'œuvre à Watteau, quels sont les arguments permettant d'y déceler une intervention du peintre Nicolas Lancret (1690-1743 $\left.{ }^{17}\right)$ ? Ce peintre, de six ans plus jeune que Watteau, est tombé sous le charme des tableaux de son aîné. Ayant commencé sa formation de peintre chez Pierre Dulin (1669-1748), il a choisi de quitter cet atelier pour rejoindre celui de Claude Gillot 


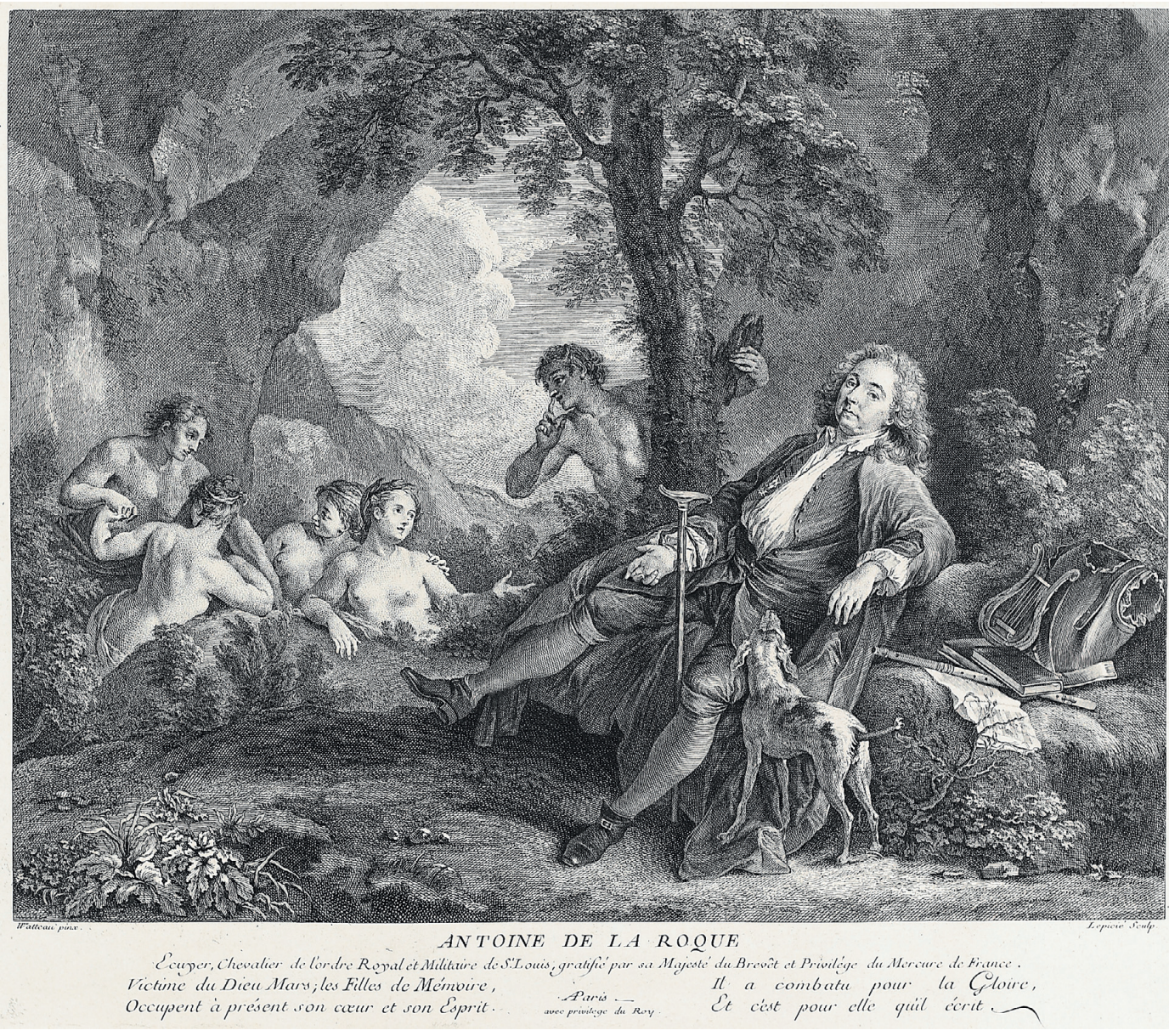

(1673-1722), le maître de Watteau, puis de solliciter son agrément à l'Académie Royale de Peinture et de Sculpture comme «peintre de fêtes galantes ${ }^{18}$. Nicolas Lancret connaît donc Watteau et son ouvre, mais aucun indice ne permet de penser qu'ils ont travaillé ensemble ${ }^{19}$. Pourtant, deux dessins de Lancret sont indiscutablement en lien avec le présent tableau ${ }^{20}$. Une Esquisse pour une Femme nue, vue de dos, aux trois crayons, conservée au musée du Louvre ${ }^{21}$, a longtemps été rangée parmi les feuilles de Watteau avant que la critique récente ne la considère comme une œuvre caractéristique de Lancret ${ }^{22}$. Il s'agit à l'évidence d'une étude préparatoire pour la nymphe de dos peinte à droite de la composition, étude qui sera réemployée par Lancret pour d'autres œuvres telles que la Diane et Callisto autrefois dans les collections des châteaux de Prusse, ou pour Les Baigneuses de l'ancienne collection $\mathrm{Bruhl}^{23}$. Un second dessin, en mains privées (fig. 3), détaille l'habillement de La Roque et en étudie séparément les deux mains ; caractéristique du style de Lancret, ce dessin est si proche du Portrait de La Roque que l'on a supposé qu'il s'agissait d'une copie dessinée par Lancret d'après le tableau ${ }^{24}$.

On connaît un exemple de gravure du Recueil Jullienne donnant à Watteau un tableau qui n'est pas entièrement de sa main. Le fameux Double portrait de Watteau et Jullienne, gravé par Tardieu en 1731 et connu sous le titre de Assis, au près de toy, sous ces charmans ombrages, est le fruit du collage d'un portrait de Watteau par lui-même, autrefois possédé par Jullienne, d'un portrait de Jullienne par François de Troy et d'une série d'études de mains dessinées par Watteau, le tout réuni artificiellement dans une composition inspirée d'une gravure de Picart, datée de $1709^{25}$. Antoine de la Roque aurait-il poussé Jullienne à attribuer à Watteau son portrait peint par Lancret? Ou bien Jullienne aurait-il encouragé son graveur François-Bernard Lépicié à ne pas faire apparaître l'apport de Lancret dans son estampe ? La petite troupe se connaissait bien : le directeur du Mercure a fait graver par Lépicié deux de ses plus beaux Chardin que Gersaint acheta à sa vente après décès ${ }^{26}$.

\section{Deux changements de format}

L'étude du dossier d'imagerie scientifique ne clarifie pas immédiatement la question des attributions, bien au contraire. La radiographie (fig. 4) montre en effet que le format actuel de l'œuvre est le fruit de deux transformations successives : elle a d'abord été peinte sur une toile rectangulaire, de petit 


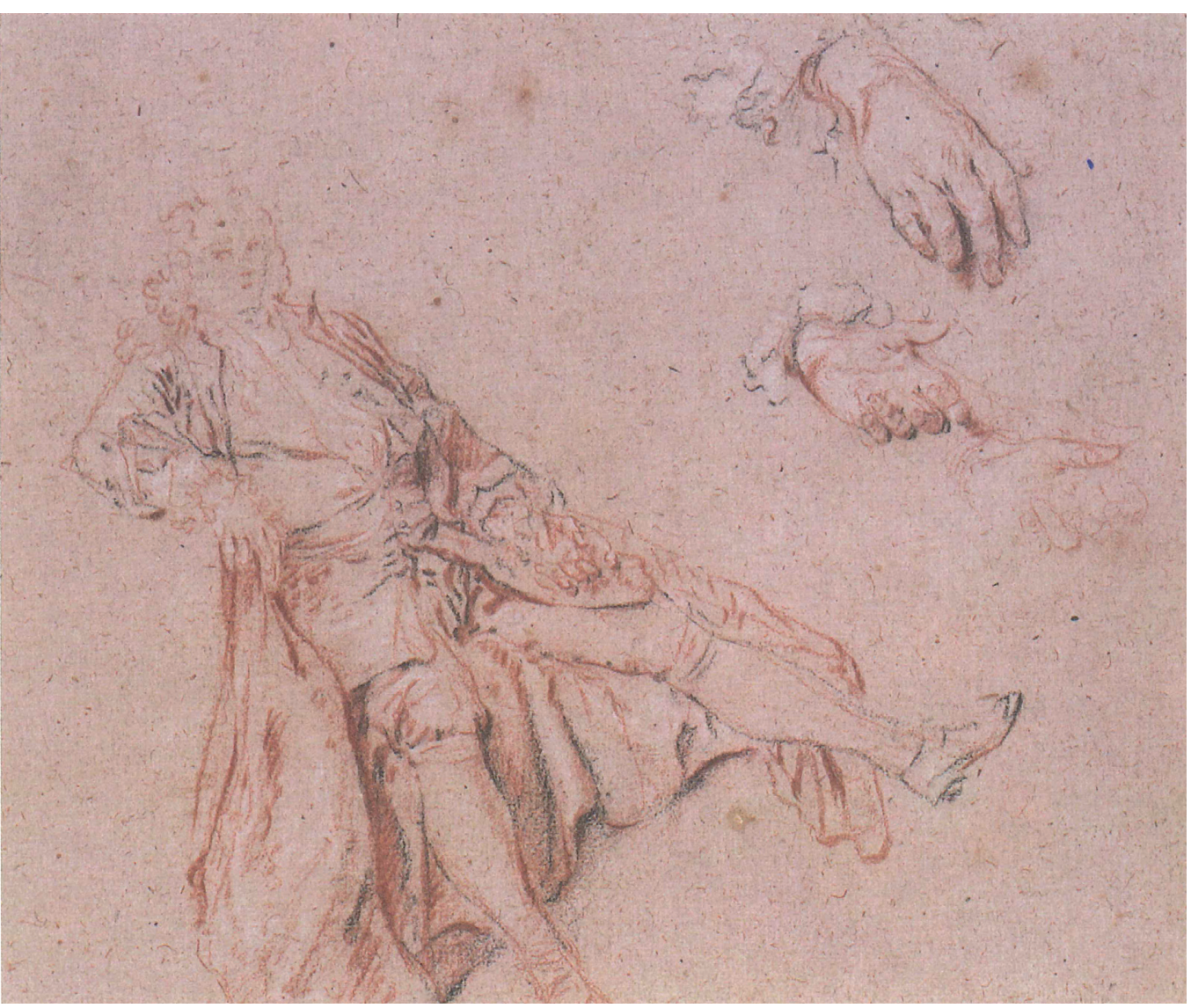

Fig. 3. Nicolas Lancret, Étude d'homme assis, la jambe gauche étendue; collection privée. (c) D.R.

format, puis a été découpée partiellement et complétée par des bandes d'agrandissement pour être mise à l'ovale, avant d'être agrandie une seconde fois pour atteindre son format actuel.

La première composition mesure $36 \mathrm{~cm}$ de haut par $42 \mathrm{~cm}$ de long. Elle est peinte sur une toile d'armure simple, à fils irréguliers, comptant environ 13 par 10 fils au cm². Aucune guirlande de tension n'est visible sur les bords, ce qui est inattendu : l'examen systématique des radiographies de tableaux de Watteau montre en effet que cet artiste peint le plus souvent sur des toiles tendues sur leur châssis définitif, comme le montre la présence de guirlandes de tension sur tous les bords ${ }^{27}$. L'absence de guirlandes sur le Portrait de La Roque peut être expliquée de deux façons : la première hypothèse est que la composition peinte par l'artiste était plus grande et ressemblait donc davantage à la gravure avant d'avoir été réduite de format ; la seconde hypothèse est que l'artiste a commencé l'exécution de l'œuvre alors que la toile était tendue sur un bâti provisoire et qu'il ne l'a mise sur son châssis définitif qu'en fin d'exécution, en découpant les bords. La première hypothèse est la plus séduisante, mais malheureusement la moins crédible. En effet, les bords latéraux et le bord supérieur de la toile sont parfaitement rectilignes ; ils ne présentent aucune irrégularité qui pourrait signaler qu'ils ont été découpés tardivement dans une toile plus grande, ni aucun signe d'accident qui aurait pu justifier ce découpage. D'autre part, cela impliquerait que le format de l'œuvre a été changé à trois reprises, et non deux, et que le format de l'œuvre a été réduit pour être agrandi presque aussitôt après afin d'être mis à l'ovale! La seconde hypothèse est la plus vraisemblable, car elle est conforme au processus de réalisation d'un portrait. En effet, un artiste commence généralement un portrait en faisant poser son modèle, dont il peint fidèlement les traits. Il peut avoir esquissé à grands traits le reste de la composition, mais guère au-delà, et se réserve souvent la possibilité de modifier la scène en travaillant sur une toile plus grande que nécessaire. Le portrait gagne d'ailleurs en présence si l'on réduit la scène (fig. 5), car l'action se concentre sur les personnages en diminuant l'importance de la falaise et du sol. Dans ce format réduit, La Roque occupe mieux le premier plan, entre le chien qui sert de repoussoir et les personnages de l'arrière-plan auxquels il est astucieusement relié par sa main gauche, qui est dirigée vers eux autant que vers sa jambe.

Pour transformer la première composition en un tableau ovale, les angles supérieurs de la toile originale ont été coupés, le bord inférieur de la toile a été arrondi et trois pièces de toile ont été ajoutées (fig. 4). La finesse des mastics placés à 
Fig. 4. Portrait d'Antoine de La Roque, radiographie. (c) C2RMF/Elsa Lambert.

Fig. 5. Portrait d'Antoine de La Roque : restitution du tableau à son format initial (d'après le cliché de Laurence Clivet).

(c) C2RMF.

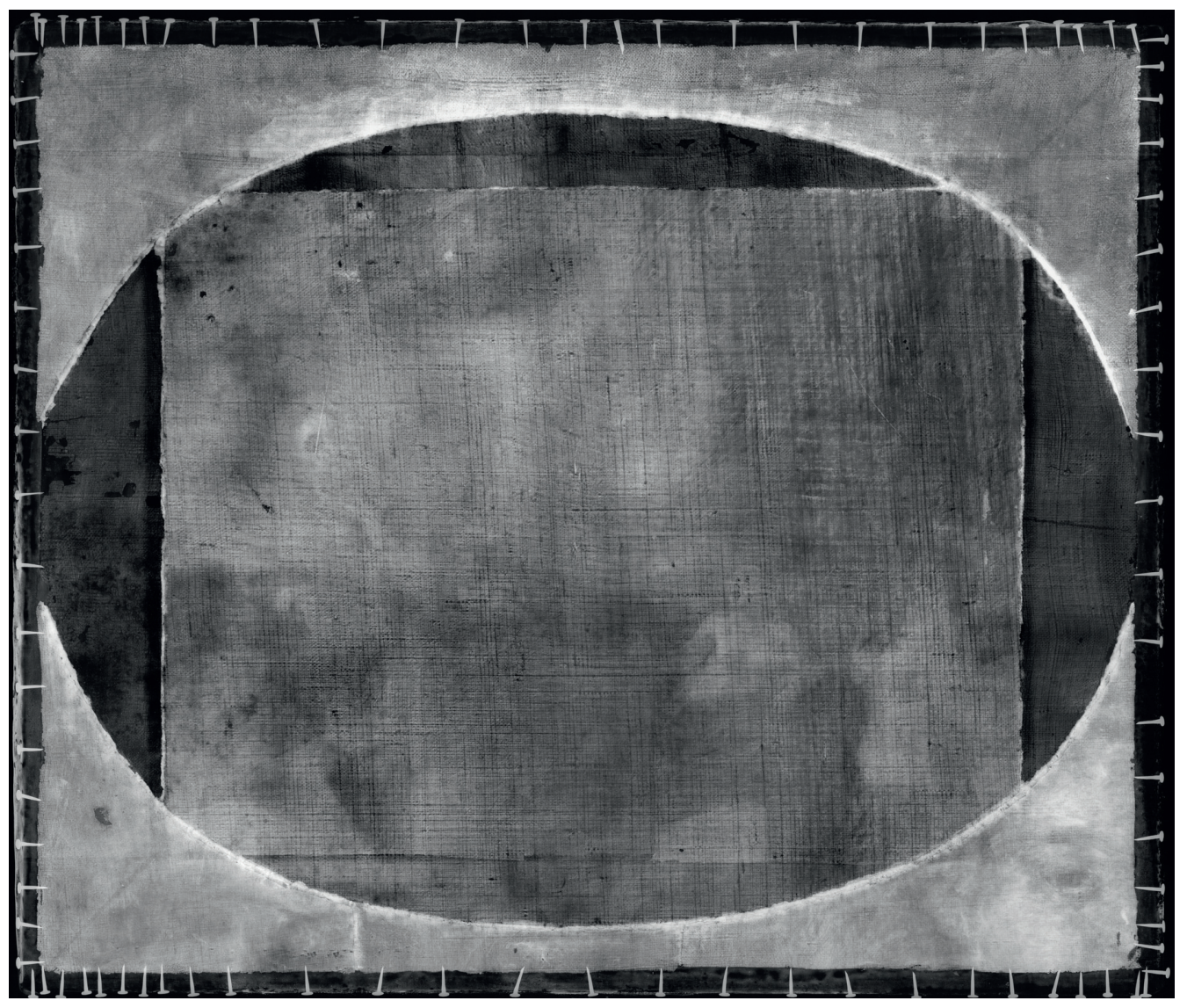


la jonction des toiles laisse soupçonner que l'assemblage a été réalisé au moyen de coutures, qui ont été arasées lors de l'agrandissement suivant. Le format obtenu est un ovale allongé, de 39,5 cm de haut par $54 \mathrm{~cm}$ de large. La toile des agrandissements est plus serrée que celle de la partie centrale (12 à 13 fils par 12 fils au cm²), tandis que la couche de préparation présente une radio-opacité différente. Une forme claire, visible sur l'agrandissement supérieur, indique que la toile est un remploi provenant d'une autre composition. La mise à l'ovale confère un ton moins grave et plus gracieux à l'œuvre, assouplissant les rythmes. L'ajout de la cuirasse lui donne une connotation militaire supplémentaire tandis que l'ouverture de la scène sur la droite met davantage en valeur le groupe des faunes et des nymphes.

Le dernier changement de format, au grand rectangle, a été fait en conservant l'existant, par rentoilage. Les parties arrondies ont été complétées par quatre morceaux de toile assujettis au reste de l'œuvre grâce à la toile collée au revers. Les toiles de complément proviennent, là encore, d'une toile de récupération car on observe un fragment de portrait d'homme dans l'angle inférieur droit. La préparation est plus radio-opaque que les autres. Avec cet agrandissement, l'œuvre est devenue identique en tous points à la gravure, ce qui n'était pas le cas auparavant.

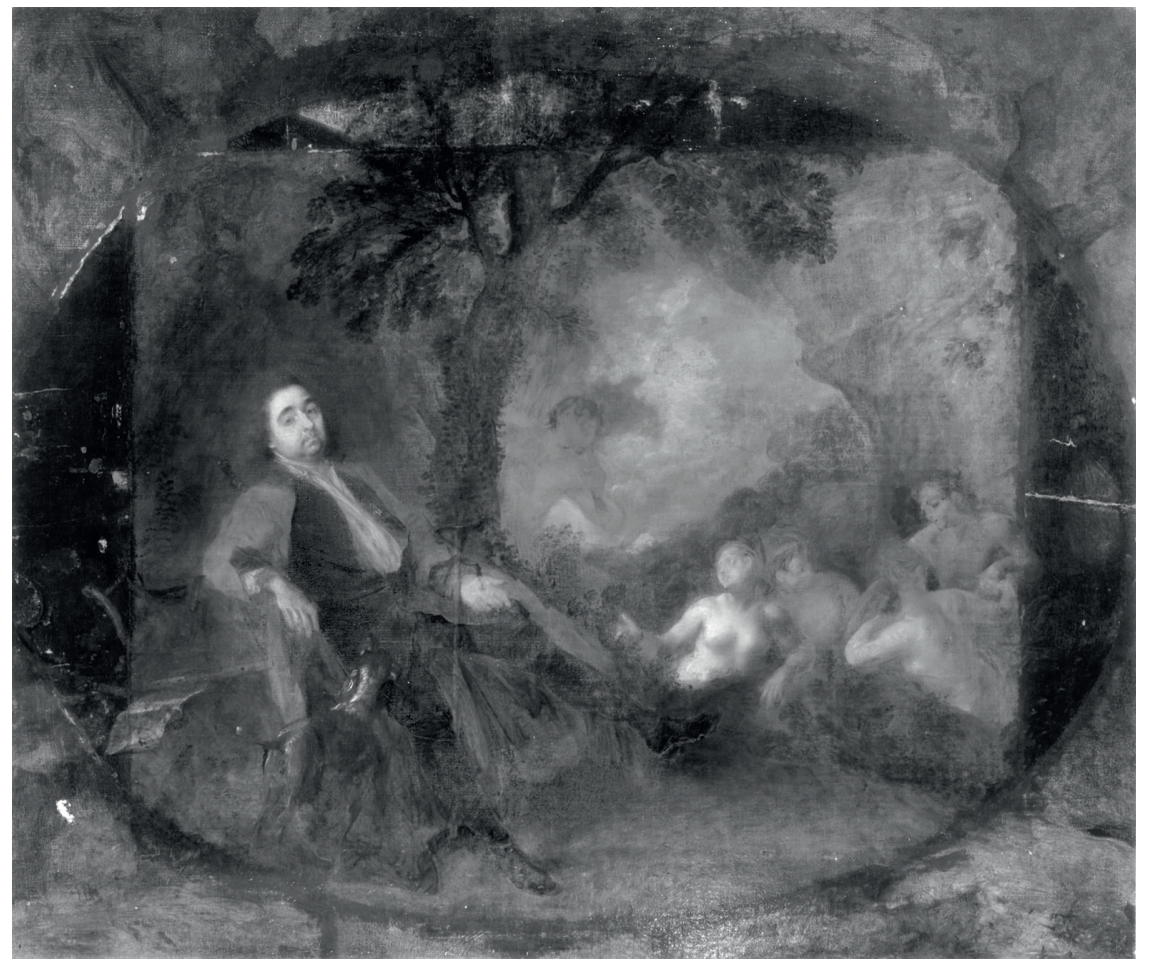

Fig. 6. Portrait d'Antoine de La Roque: réflectographie infrarouge. (C) C2RMF/Jean-Louis Bellec.

\section{La préparation et le dessin sous-jacent}

Aucun prélèvement de matière n'a été réalisé, mais une analyse par spectrométrie de fluorescence $\mathrm{X}$ a permis d'observer que la préparation de la partie centrale est à base de blanc de plomb et d'ocres, dont la couleur rose clair s'observe dans les parties un peu usées de la chevelure de La Roque ou autour de l'œil de la nymphe vue de face. Cette préparation rose recouvre très probablement une première préparation de couleur rouge. Celle-ci n’a pas été observée directement mais est traditionnelle aux peintures françaises du début du XVIII ${ }^{\mathrm{e}}$ siècle ; elle est fréquente sur les ouvres de Watteau et de son entourage ${ }^{28}$ tandis qu'aucune n'a été repérée à ce jour chez Lancret. On se gardera toutefois de tirer des observations hâtives sur ce point, en l'absence de tout prélèvement.

Le premier agrandissement, à l'ovale, est peint sur une sous-couche brune de tonalité plus froide que la partie centrale. Probablement étendue au-dessus d'une composition sous-jacente, cette sous-couche contient moins de blanc de plomb, plus de fer et de cuivre qu'en partie centrale ; elle est donc différente de cette dernière, mais ne peut être datée précisément car sa coloration et ses matériaux se rencontrent continûment du XVII ${ }^{\mathrm{e}}$ siècle au XIX ${ }^{\mathrm{e}}$ siècle.

L'agrandissement au rectangle est couvert d'une souscouche gris-beige qui cache la composition peinte au-dessous. Elle contient une importante quantité de blanc de plomb, ce qui correspond à une formulation que l'on trouve à partir de la fin du XVIII ${ }^{\mathrm{e}}$ siècle et tout au long du XIX ${ }^{\mathrm{e}}$ siècle.

Le dessin de mise en place de la composition, appelé dessin sous-jacent, n'est observable que partiellement en réflectographie infrarouge (fig. 6). Quelques traits de mise en place, posés au pinceau, précisent le pourtour de la jambe gauche et de la robe, tandis que d'autres traits structurent le bas du manteau. Ces tracés ne doivent pas être confondus avec les lignes que l'on observe autour d'une main de l'une des nymphes ou au pourtour de l'épaule du faune debout, qui ont vraisemblablement été tracées par un restaurateur afin de redéfinir les formes dans des parties abrasées. Le document apporte aussi une information essentielle sur la mise en œuvre de la peinture car il montre que le corps de La Roque a été entièrement peint avant d'être recouvert 
Fig. 7. Portrait d'Antoine de La Roque : photographie infrarouge en faussescouleurs. (C) C2RMF/ Laurence Clivet.

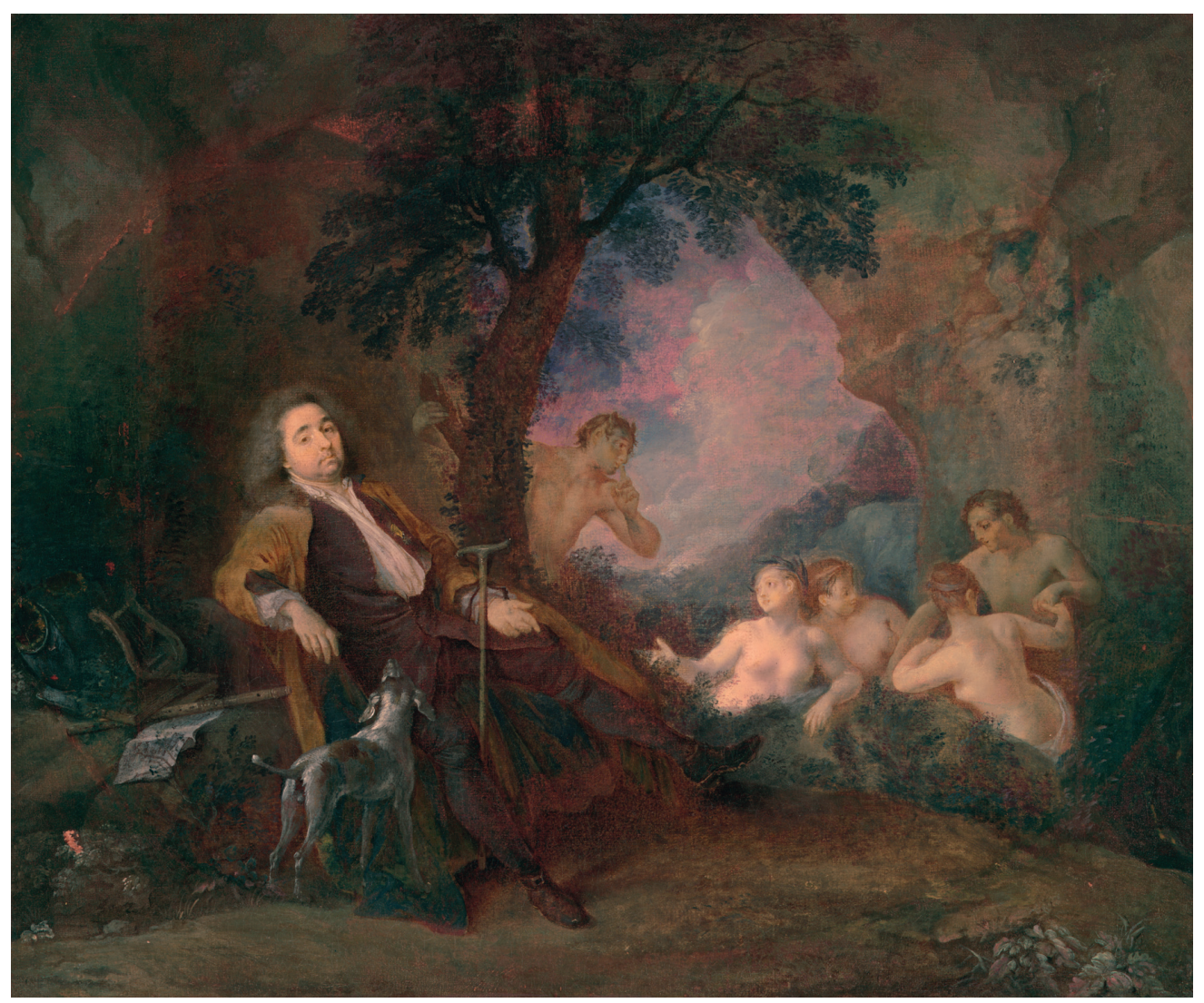

en partie par le chien et par la canne du modèle. Cette observation suffit à démontrer que l'œuvre est bien un original et non une copie réalisée d'après une œuvre disparue, car le copiste aurait laissé les emplacements du chien et de la canne en réserve. La photographie infrarouge en fausses-couleurs confirme que la doublure sombre du manteau de La Roque a été entièrement peinte avant d'être cachée par le chien (fig. 7).

Le manteau que l'on observe sur la réflectographie infrarouge est très comparable au manteau dessiné par Lancret sur le dessin conservé en collection privée (fig. 3). Ce dessin ne comporte ni la canne, ni le chien. Il met en place les détails du costume à l'identique de la peinture, notamment dans le négligé du boutonnage et dans la position des mains. Une ligne en accolade tracée au fusain au-dessus du poignet gauche indique même l'emplacement de la dragonne qui relie la canne à la main. On remarque que ce dessin ne s'attarde pas à la représentation des traits du modèle, qui semble plus jeune qu'Antoine de La Roque. Il s'agit probablement une étude de costume et de mains, qui a été posée par un assistant afin de compléter le visage pour lequel La Roque avait lui-même posé. Si l'on admet que Lancret est bien l'auteur du dessin, il faut en déduire que cette œuvre n'est pas une copie d'après la peinture, mais peut-être une copie d'après un dessin perdu d'Antoine Watteau, hypothèse improbable, ou encore un dessin original de Lancret qui devient alors l'auteur présumé de cette partie du tableau. Rappelons que Lancret est vraisemblablement l'auteur du groupe de faunes et de nymphes peint à l'arrière-plan, dont on conserve un dessin préparatoire de sa main. L'exécution peinte du costume de La Roque est au demeurant assez faible, les mains sont molles, peu structurées, bien éloignées des mains allongées et osseuses que Watteau construit par juxtaposition de hachures; le vêtement n'a pas le chatoiement des tissus du maître, tandis que le chien est bien éloigné des animaux pleins de vie que Watteau affectionne : tout indique que le corps de La Roque n'est pas de Watteau mais de Lancret.

Lancret semble donc l'auteur d'une grande partie de la composition centrale. En revanche, il n'est pas l'auteur des agrandissements. La réflectographie infrarouge montre en effet que la partie haute de l'arbre a été peinte avec une matière plus fine que la composition centrale et que le tronc n'est pas correctement raccordé à la partie basse. Le bras du faune de droite est trop épais par rapport au raccourci de l'avant-bras, le complément de lyre est plus opaque que le reste de l'instrument, tandis que les pages de la partition ne respectent pas la disposition du texte imprimé. L'agrandissement de mise à l'ovale n'est donc pas en continuité avec la scène et ne peut, de ce fait, être attribué à Lancret. L'agrandissement final, de mise au rectangle, est encore plus décevant : il s'agit d'une peinture d'accompagnement, peu élaborée picturalement. 


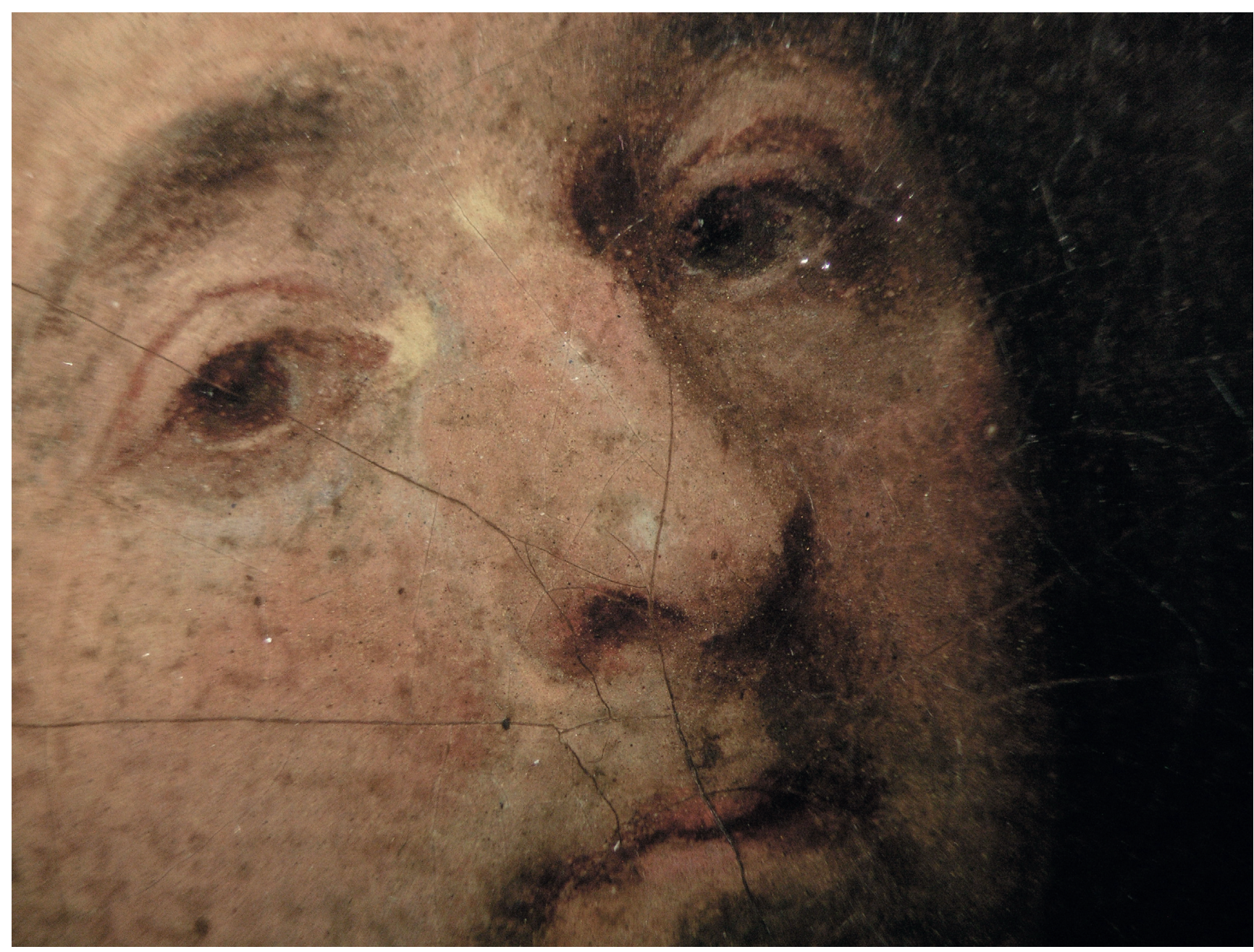

Fig. 8. Portrait d'Antoine de La Roque, détail du visage, lumière directe (C) C2RMF/Bruno Mottin.

\section{Un visage peint par Watteau?}

Le corps de La Roque présente donc des faiblesses d'exécution qui contrastent avec la qualité du traitement du visage. Malgré sa taille de miniature, l'artiste a construit savamment le rendu des traits à partir d'une sous-couche grise, que l'on observe sur les joues et autour de l'œil droit, sur laquelle il a déposé une succession de petites touches roses, rougeâtres, brunes, jaune vif ou beige clair qui structurent et donnent vie aux chairs (fig. 8). Le visage de La Roque s'attache maladroitement au cou, qui est peint avec une matière épaisse et fripée : le modèle n'a donc posé que pour la tête. Les faunes et les nymphes ne sont pas exécutés avec la même subtilité. Watteau pourrait donc n'avoir peint que la tête.

Pour tenter de préciser cette question, nous avons demandé à Thomas Calligaro, chercheur au C2RMF, de réaliser une cartographie d'une partie du tableau par spectrométrie de fluorescence $\mathrm{X}$, méthode récemment implémentée au C2RMF ${ }^{29}$. L'appareil de mesure a été monté sur un bras mobile et programmé pour réaliser des pointés réguliers sur la surface, millimètre par millimètre, dans une zone de 30 cm de large par $9 \mathrm{~cm}$ de haut. La zone sélectionnée a compris le visage de La Roque, son cou, les bandes d'agrandissement de gauche, le faune debout et une partie du ciel. Nous avons ainsi obtenu une série d'images signalant la présence d'environ 13 éléments chimiques et permettant d'identifier, par déduction, la présence de nombreux pigments ${ }^{30}$. L'interprétation des résultats est toutefois délicate, des éléments comme le plomb répondant fortement et pouvant cacher d'autres éléments. En effet, la réponse obtenue varie en fonction de l'énergie des rayons X émis et de sa profondeur au sein de la stratigraphie de la couche peinte.

Les images montrent que l'œuvre a été assez endommagée par un ancien rentoilage et que des couleurs comme le brun, ou même le ciel, ont dû être harmonisées par des repeints. L'abondance de zinc dans le ciel et sa présence dans les ombres brunes signalent des retouches dans ces zones (fig. 9a). Les parties non retouchées du ciel sont vraisemblablement à base de bleu de Prusse, pigment qui n'est pas détectable par fluorescence $\mathrm{X}$ mais qui conserve une couleur bleue sous 
infrarouge fausses-couleurs (fig. 7). Ce pigment se rencontre fréquemment dans les dernières œuvres de Watteau ainsi que chez ses émules. Dans les nuages, la couleur rose sous infrarouge fausses-couleurs signale quelques retouches à base de zinc, mais peut-être aussi l'emploi d'indigo, pigment que Watteau utilise souvent en sous-couche (le Pèlerinage, le Pierrot), alors qu'il n'a pas encore été repéré chez ses suiveurs.

La présence de fer et de potassium dans les verts indique la présence de terre verte, pigment que Watteau et Lancret emploient tous deux. Un peu de manganèse signale l'utilisation de terre d'ombre. Le visage de La Roque est peint avec un mélange de blanc de plomb (fig. 9b) additionné de vermillon (fig. 9c), d'un peu d'antimoine qui signale la présence de jaune de Naples (fig. 9d), et peut-être d'un peu de noir de carbone, non détectable par la méthode employée. Le cou contient moins de plomb, un peu de fer (fig. 9e) et beaucoup de jaune de Naples, ce qui explique son ton plus vif. Cette observation confirme que le cou et la tête n'ont pas été peints ensemble. La carnation du faune ne contient pas de vermillon, mais des ocres indiquées par la présence de fer, avec une plus grande proportion d'antimoine. Or, les études menées sur les tableaux de Watteau ont montré qu'il emploie presque toujours du vermillon dans ses carnations alors que Lancret ou Pater ne l'utilisent que rarement et préfèrent les ocres ${ }^{31}$ : l'observation va à nouveau vers l'attribution du seul portrait à Watteau.

Les pigments employés pour la mise à l'ovale sont différents de ceux de la partie centrale. Ils contiennent davantage de cuivre, de fer et de manganèse, alors que le blanc de plomb est moins présent. Ces pigments sont traditionnels et suggèrent que le premier agrandissement est antérieur à la révolution chimique des années 1830. Le dernier agrandissement est difficile à dater par ses pigments, mais la couche peinte a été vieillie artificiellement au moyen de fausses craquelures, tracées à l'aiguille, dont le sillon a creusé irrégulièrement la préparation.

\section{Conclusion : un tableau à quatre mains ?}

Nous pouvons à présent retracer avec davantage de certitudes l'histoire complexe de ce tableau, sans en dissiper toutes les ombres. Antoine de La Roque a commandé son portrait à un artiste et a posé quelques heures devant lui. Cet artiste est probablement Watteau, si l'on se base sur la lettre de la gravure qui lui attribue l'œuvre, ainsi que sur la technique fine et sur le rendu psychologique aigu des traits du visage. L'œuvre étant restée inachevée, dans un format au petit rectangle, La Roque a demandé à Nicolas Lancret de terminer ce portrait. Comme le corps d'Antoine de La Roque n'était pas assez précisé sur l'ébauche, Lancret a fait poser un assistant pour mettre au point l'agencement des vêtements et pour étudier les mains. Il a peint le corps d'après le dessin, sans la canne et le chien qu'il a ajoutés par la suite. Il a également peint les autres figures et le paysage de l'arrière-plan, en s'appuyant peut-être sur une sous-couche bleue peinte à l'arrière-plan
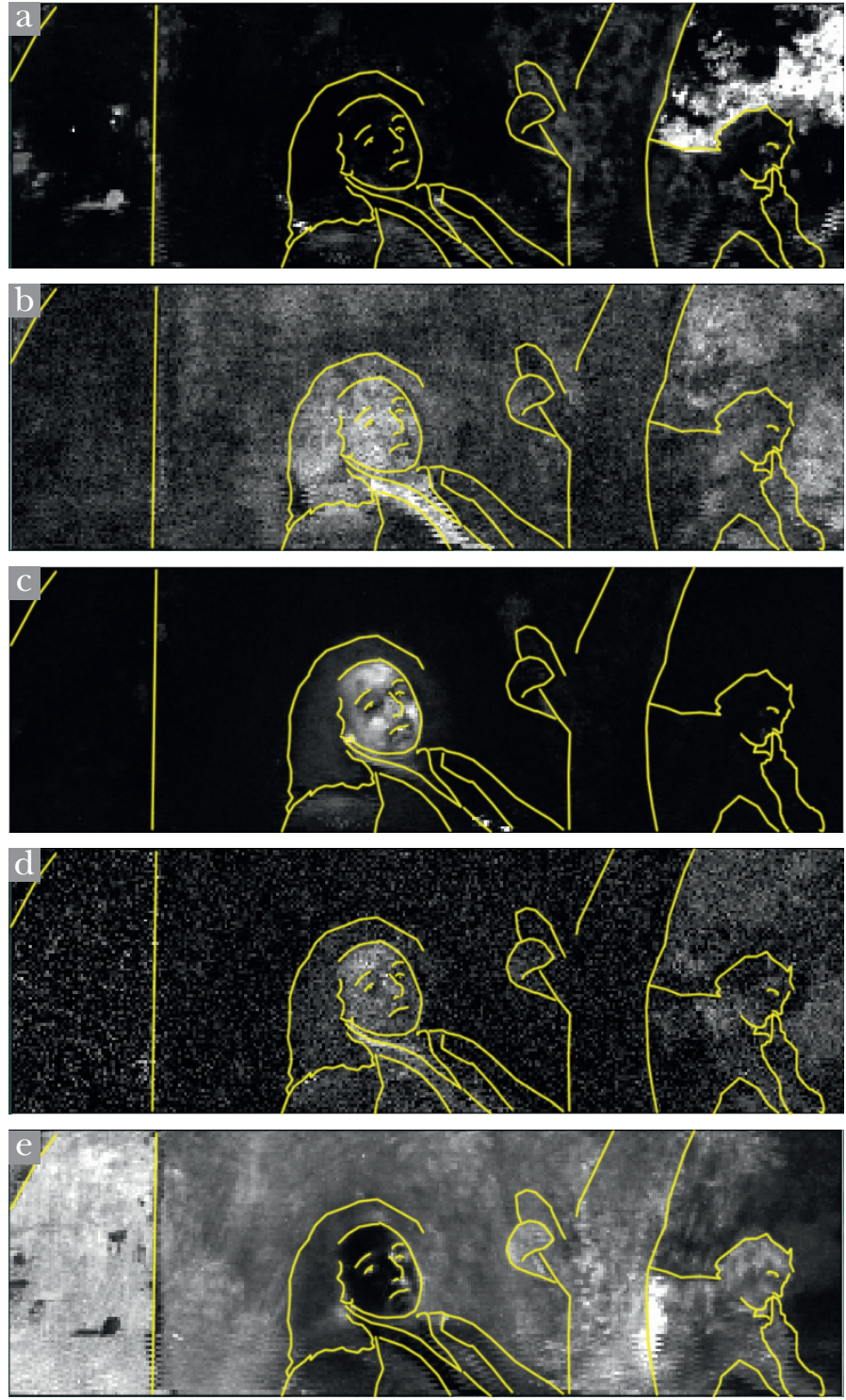

Fig. 9. Portrait d'Antoine de La Roque, images du zinc (a), des raies M du plomb (b), du vermillon (c), de l'antimoine (d) et du fer (e) par spectrométrie de fluorescence X. Les contours de la composition ont été indiqués en jaune. (c) C2RMF/Thomas Calligaro. 
par Watteau, comme pourrait l'indiquer la présence supposée d'indigo. La part de Watteau dans la conception de ce tableau s'arrêterait donc au portrait, Lancret étant responsable de la plus large part, ce qui explique pourquoi il a réutilisé l'attitude de La Roque sur deux autres portraits ${ }^{32}$.

Que la composition centrale soit une ouvre à deux mains n'a rien de surprenant. Gersaint en mentionne plusieurs dans le catalogue de la vente Laroque ${ }^{33}$ : Lancret a peint les figures des tableaux de Saftleven et a repris des figures de tableaux de Kalf ${ }^{34}$. On sait aussi que Watteau a ajouté un couple dans un paysage de Francisque II Millet ${ }^{35}$ et que Crozat possédait deux tableaux de Jean-Baptiste Forest « où les figures sont peintes par Watteau ${ }^{36}$ ». Nicolas Lancret a copié certaines œuvres de Watteau : la composition qui a servi de modèle à qui a jugé bon d'ajouter une série de tableaux en partie haute afin d'améliorer le format de l'original ${ }^{37}$. Cet artiste a-t-il peint une seconde version du Portrait d'Antoine de La Roque, plus aérée, pour servir de modèle à la gravure ?

Faute de pouvoir dater précisément le premier agrandissement, il est difficile de préciser s'il a été fait avant la gravure et lui a servi de modèle, ou bien si la gravure a été utilisée pour la mise à l'ovale. Il est en revanche certain que la gravure a été fidèlement copiée dans la seconde moitié du XIX ${ }^{\mathrm{e}}$ ou au début $\mathrm{du} \mathrm{XX}^{\mathrm{e}}$ siècle, pour mettre la composition au format d'un grand rectangle qui n'a probablement jamais existé sur l'original. Le Portrait d'Antoine de La Roque se présente donc aujourd'hui comme le résultat de quatre étapes distinctes, réalisées par quatre mains ! (1656-1722) et publiée en 1732 dans le Recueil Jullienne ne s'inspire pas de la peinture originale, pourtant déjà mise au rectangle, mais d'une version en petit, copiée par Lancret

\section{Notes}

1. Portrait d'Antoine de la Roque, vers 1715 ?, Tokyo (Japon), Fuji Art Museum (Inv. 530 AB 020).

2. Le tableau ne figure pas dans la vente La Roque de 1745 (catalogue par Gersaint). Il est probablement resté dans la famille du modèle, car il est signalé en 1921 au château de Vézenobres, propriété des Bernis-Calvières, apparentés à La Roque. Il est acheté par le marchand André Weil, qui le revend vers 1925 à la collection Levy, de Strasbourg (Camesasca, 1982). Il passe ensuite dans la collection Roberto Polo, puis est acheté à sa vente par le Tokyo Fuji Art Museum (Paris, 30 mai 1988, n 21). Expositions : Bruxelles, exposition internationale, 1935, $\mathrm{n}^{\circ} 978$; New York, 1954, n 31 ; Stockholm, 1964, n 4 ; Johannesburg, 1974, sans $n^{\circ}$; Atlanta, 1983, n 23 ; Séoul, 1990, sans n ; Bruxelles, 2013 $\mathrm{n}^{\circ} 141$.

3. Watteau, 1684-1721, 1984-1985, p. 192, D. 113 .

4. Raymond F. (dir.), 2013, cat. 141

(tableau) et 142 (gravure), p. 242-243.

5. Watteau et la fête galante, 2009-2010.

6. Nous remercions tout

particulièrement M. Akira Gokita, directeur du Tokyo Fuji Art Museum qui a bien voulu autoriser cette étude, ainsi que Ken Okamura, Yoshiki Ono, Maki Koudedji. Nous remercions également les équipes du Palais des Beaux-Arts de Bruxelles (BOZAR) : Paul Dujardin, Sophie Lauwers et Roció del Casar, les équipes du C2RMF : Laurence Clivet, Jean-Louis Bellec, Elsa Lambert, Eric Laval, Myriam Eveno, ainsi que les nombreuses personnes avec qui nous avons discuté de notre enquête : Guillaume Faroult, Corine Le Bitouzé, Elisabeth Martin, Pierre Rosenberg, Marie-Catherine Sahut, Mary Tavener Holmes et Christoph Vogtherr.

7. La date du portrait est discutée : Clément de Ris, dans les Amateurs d'autrefois (1877, p. 225) considère arbitrairement qu'il s'agit de la dernière œuvre de Watteau ; Dacier et Vuaflart (DV 269) refusent de placer ce portrait avant 1718 ; les dates proposées par les historiens contemporains varient de 1712 (Mathey) à 1716 (Adhémar), tandis que Cailleux (1964) propose la période 1713-1715 en raison de la présence de livrets d'opéra sur le tableau. Ces opinions assez difficilement vérifiables vont à l'encontre de la datation tardive, vers 1720 proposée récemment pour l'étude du corps de La Roque par Nicolas Lancret

(Wintermute, 1999).

8. Sur La Roque, voir notamment Moureau F., 2001.

9. Abbé Fraguier, tombeau poétique de Watteau, placé en tête des Figures de Différents Caractères.

10. Rosenberg P., 1984.

11. Portrait présumé de La Roque, fusain sur papier, 228 x 169 mm, Cambridge, Fitzwilliam Museum, inv. 2266 (RosenbergPrat, $n^{\circ} 317$ ).

12. Antoine de La Roque, « Les Beaux Arts ", Le Mercure, août 1721, p. 81-83.

13. Sahut M.-C., Raymond F., 2010.

14. Gersaint E.-F., 1745. Les trois tableaux sont Les Fatigues et Les délassements de la Guerre (deux tableaux format pendant, $\mathrm{n}^{\circ} 44$ du catalogue de vente, aujourd'hui à
Saint-Pétersbourg, musée de l'Ermitage), et un Saint François ( ${ }^{\circ} 149$, perdu).

15. Euvres / Des Estampes Gravées / d'après / Les Tableaux Ẽ Desseins / de feu Antoine / Watteau / peintre flamand / de l'Académie Royale / de Peinture Ẽ de Sculpture. / Quatrième Ẽ dernière Partie, 1734, folio 91. Lépicié n'a gravé pour Jullienne que le Portrait de La Roque et quatre Figures de Différents Caractères (folios 99, 129, 224, 342). Il grava aussi pour Odieuvre, en 1736, le fameux Autoportrait de Watteau (ancienne collection Jullienne).

16. Le Mercure, juin 1734, t. II, p. 1405. Inventaire du Fonds Français, Graveurs du XVIII siècle, 1977, tome 14, p. 364 et 386. La gravure est annoncée à la vente chez Surugue et chez la veuve de F. Chéreau par Le Mercure, janvier 1735, p. 122.

17. Holmes M. T., 1991

18. Watteau est agréé à l'Académie en 1712, et reçu en 1717 comme " peintre d'histoire "; Lancret est agréé en 1718 et reçu en 1719 comme " peintre de fêtes galantes" (C. Michel, 2008, p. 172-180).

19. Aucune biographie de Watteau ne mentionne de collaboration avec Lancret. Seul Dezallier d'Argenville qualifie Pater et Lancret de " disciples de Watteau » (Rosenberg, 1984, p. 51). Quant à la biographie de Lancret écrite par Ballot de Sovot, elle insiste sur le lien entretenu entre les deux artistes au début de la carrière de Lancret sans toutefois indiquer de réalisations conjointes (Guiffrey J., 1874, p. 19). Martin Eidelberg a toutefois émis récemment l'hypothèse que Nicolas Lancret était un assistant rémunéré de Watteau lorsque ce dernier peignait le portrait 
de La Roque (The Young Lancret and Watteau,http://watteauandhiscircle.org/ younglancret.htm, février 2015, modifié en mars 2015).

20. L'exemplaire du Catalogue raisonné de l'œuvre peint, dessiné et gravé d'Antoine Watteau par Edmond de Goncourt (Paris, 1875), conservé au Cabinet des estampes de la Bibliothèque nationale, présente des annotations troublantes de la main de l'auteur : $\mathrm{n}^{\circ} 17,:$ « Le portrait peint d'Antoine de La Roque (H. 98 c. ; L. 60 c.) se vendait $1700 \mathrm{fr}$, sous le $\mathrm{n}^{\circ} 894$ à la vente du lieutenant Despinoy, en 1850. Un croquis de cette composition avec des différences est dans la collection de M. Carrier. " Note manuscrite : « ce croquis se vendait ... à sa vente (1875). Il était accompagné de deux académies de femmes pour les naïades, le directeur du Mercure. Ces dessins étaient assez douteux. » Les dimensions données par Goncourt ne sont pas celles du tableau étudié. Erreur ou preuve de l'existence d'une autre version ? Les dessins " douteux " mentionnés seraient-ils ceux de Lancret? Les recherches relatives à la vente Carrier (1875) ne peuvent confirmer ou infirmer à l'heure actuelle cette hypothèse qui reste d'importance. La version conservée au musée de La Fère (H. 72 ; L. 92 cm) issue, d'après l'historique, de la vente Despinoy, ne présente pas les dimensions indiquées par Goncourt (à moins que celles-ci aient été inversées en nichant des erreurs?).

21. Femme nue, vue de dos à mi-corps, 102 x 109 mm, Paris, musée du Louvre, département des arts graphiques Inv. Rec 89, recto ; fonds de la Commission de récupération artistique.

22. L'œuvre est rejetée du corpus des dessins de Watteau (Rosenberg P., Prat L.-A., 1996, n R78) ; elle est publiée sous le nom de Lancret par Holmes M.T., dans Vogtherr C.-M., 2011, p. 476, ainsi que par Raymond F., 2013., ill.46.

23. Nicolas Lancret, Diane et Callisto, 58 x 71, œuvre disparue en 1945 (Holmes M. T., in Vogtherr C., 2011, cat. $\mathrm{n}^{\circ} 52$ ) ; Nicolas Lancret, Les Baigneuses de Lancret, ancienne collection du comte de Bruhl, 1754 (Wildenstein G., 1924, $\mathrm{n}^{\circ}$ 429, pl. 105). 24. Eidelberg M., 2000, p. 62-63. 25. Vogtherr C.-M., Tonkovich J., 2011, p. $72-82$.

26. Gersaint E.F., $1745, \mathrm{n}^{\circ} 190$

27. On observe des guirlandes de tension autour du Portrait de Gentilhomme (Paris, Louvre, RF 1973-1), de l'Automne, ovale peint sur un toile rectangulaire (Paris, Louvre, MI 1129), de la Diane au bain (Paris, Louvre, RF 1977-447), du Pèlerinage à l'âle de Cythère (Paris, Louvre, Inv. 8525), du FauxPas (Paris, Louvre, MI 1127), de la Nymphe et Satyre (Paris, Louvre, MI 1129), et de la Déclaration attendue (Angers, musée des Beaux-arts, MBAJ 182), ces deux derniers ovales peints sur une toile rectangulaire.

28. Martin E., Sindaco-Domas C., 20092010, n 30-31, p. 27-36; Most M., 2011, p. $96-106$.

29. Eveno M., Ravaud R., Calligaro T., Pichon L., Laval E., 2014.
30. Les principaux éléments chimiques repérés sont le baryum, le calcium, le chrome, le cobalt, le cuivre, le fer, le mercure, le potassium, le manganèse, le plomb (raies L et M), l'antimoine, le titane et le zinc.

31. Eveno M., Laval L., Martin, E., Bartoll J., 2009-2010, p. 41.

32. Le Chasseur au repos (anciennement collection Forsyth Wickes) ; la Fin de la Chasse (anciennement collection Tabourier), publiés par Cailleux J., 1964 ; voir aussi Holmes M. T., 1991, cat. 15, pl. 19.

33. Gersaint E. F., 1745, $\mathrm{n}^{\circ} 55$.

34. Banks O., 1977, p. 126-127; Glorieux G., 2002, p. 403 ; Michel C., 2008, p. 139. Les tableaux se trouvent aujourd'hui à l'Ermitage de Saint-Petersbourg (Wildenstein G., 1924, n 529-530) et à l'Art Museum de Saint-Louis, où le travail de Lancret a été effacé (Eidelberg M., 2001 p. 31). Une huile sur bois conservée à la Wallace Collection de Londres, Jeune fille dans une cuisine est peinte par Lancret sur un fond hollandais.

35. Antoine Watteau et Francisque II Millet, Le Flûtiste, vers 1716-1717, huile sur toile, Grenoble, Musée des Beaux-Arts, Inv. MG 186 (Eidelberg M., 1969).

36. Signalé par Mariette lors de la vente Crozat de 1751 (Stuffmann M., 1985, p. 109, n429-430; C. Michel, 2008, p. 139).

37. Vogtherr C-M., 2007, p. 236-304.

La copie de Lancret d'après l'Enseigne de Gersaint est aujourd'hui dans une collection particulière, Suisse.

\section{Bibliographie}

Banks O., 1977, Watteau and the North. Studies in the Dutch and Flemish Baroque Influence on French Rococo Painting, Garland, New York-Londres.

Cailleux J., 1964, « L’Art du Dix-huitième Siècle : Invalids, Huntsmen and Squires ", The Burlington Magazine, advertisement supplement, vol. 106, n731, février, p. I-III.

Eidelberg M., 1969, "The Painters of the Grenoble 'Flutist' and Some Other Collaborations", The Art Quarterly 32, p. 282-291.

Eidelberg, M., 2000, "Exhibition Reviews Watteau and His World: French Drawing from 1700 to 1750 ", The Burlington Magazine 142, janvier, p. 62-63.

Eidelberg M., 2001, "The Case of the Vanishing Watteau", Gazette des BeauxArts, 6, 138, juillet-août, p. 31.

Eveno M., Laval L., Martin, E., Bartoll J., 2009-2010, « La palette de Watteau et de ses épigones : l'analyse des pigments ", dans Watteau et la fête galante, Technè, $\mathrm{n}^{\circ}$ 30-31, p. 37-51.

Eveno M., Ravaud R., Calligaro T., Pichon L., Laval E., 2014, "The Louvre Crucifix by Giotto. Unveiling the original decoration by 2D-XRF, X-ray radiography, Emissiography and SEMEDX analysis”, Heritage Science, 2 (2014) p. 17.
Gersaint E.-F., 1745, Catalogue raisonné des différents effets curieux et rares contenus dans le Cabinet de feu M. le Chevalier de La Roque, Barois et Simon, Paris.

Glorieux G., 2002, À l'enseigne de Gersaint, Edme-François Gersaint, marchand d'art sur le pont Notre-Dame (1694-1750), Champ-Vallon, Seyssel.

Guiffrey J., Éloge de Lancret par Ballot de Sovot, Baur-Rapilly, Paris, s.d. [1874].

Holmes M. T., 1991, Nicolas Lancret, 1690-1743, Abrams, New York.

Martin E., Sindaco-Domas C., 2009-2010, «La Technique picturale des peintures de fêtes galantes dans le contexte du XVIIIJ siècle ", dans Watteau et la fête galante, Technè, $\mathrm{n}^{\circ}$ 30-31, p. 25-36.

Michel C., 2008, Le "Célèbre Watteau », Droz, Genève.

Most M., 2011, « Zur Maltechnik von Nicolas Lancret », dans Vogtherr C.-M. (dir.), p. $96-106$.

Moureau F., 2001, «De Watteau à Chardin : Antoine de La Roque, journaliste et collectionneur ", Mélanges en hommage à Pierre Rosenberg, Réunion des musées nationaux, Paris, p. 349-355.

Raymond F. (dir.), 2013, Antoine Watteau (1684-1721), la Leçon de musique, 2013, cat. exp., Bruxelles, Palais des BeauxArts, Flammarion, Paris.

Rosenberg P., 1984, Vies anciennes de Watteau, Hermann, Paris.

Rosenberg P., Prat L.-A., 1996, Antoine Watteau, 1684-1721 ; catalogue raisonné des dessins, Leonardo Arte, Milan.

Watteau et la fête galante, 2009-2010, «Watteau et la fête galante. Études techniques sur la peinture française de la première moitié du XVIII ${ }^{\mathrm{e}}$ siècle " (éd. par Sahut M.-C.et Volle N., avec la collaboration de Raymond F.), Technè, $\mathrm{n}^{\circ}$ 30-31.

Sahut M.-C., Raymond F., 2010, Antoine Watteau et l'art de l'estampe, Musée du Louvre, Paris.

Stuffmann M., 1985, "Jean Antoine Watteau (1684-1721), Die Einschiffung nach Cythera ", Berlin.

Tillerot I., 2010, Jean de Jullienne et les collectionneurs de son temps : un regard singulier sur le tableau, Maison des Sciences de l'Homme, Paris.

Vogtherr C.-M., 2007, "Watteau 'Shopsign' : The Long Creation of a Masterpiece", The Burlington Magazine, vol. 149, $\mathrm{n}^{\circ} 1250$, p. 236-304.

Vogtherr C.-M. (dir.), 2011, Französische Gemälde I, Watteau, Pater, Lancret, Lajoüe, Stiftung Preussische Schlösser und Gärten, Berlin-Brandenbourg, Akademie Verlag, Berlin.

Vogtherr C.-M., Tonkovich J., 2011, Jean de Jullienne, Collector and Connoisseur, cat. exp. Londres, Wallace Collection

Watteau (1684-1721), 1984-1985, cat. exp., Washington, National Gallery of Art, Paris, Grand Palais, Charlottenbourg, château, Réunion des musées nationaux, Paris.

Wildenstein G., 1924, Lancret, Servant, Paris. 